\title{
Multi-Agent Deep Reinforcement Learning using Attentive Graph Neural Architectures for Real-Time Strategy Games
}

\author{
Won Joon Yun ${ }^{1}$, Sungwon $\mathrm{Yi}^{2}$, and Joongheon $\mathrm{Kim}^{1}$
}

\begin{abstract}
In real-time strategy (RTS) game artificial intelligence research, various multi-agent deep reinforcement learning (MADRL) algorithms are widely and actively used nowadays. Most of the research is based on StarCraft II environment because it is the most well-known RTS games in worldwide. In our proposed MADRL-based algorithm, distributed MADRL is fundamentally used that is called QMIX. In addition to QMIX-based distributed computation, we consider state categorization which can reduce computational complexity significantly. Furthermore, self-attention mechanisms are used for identifying the relationship among agents in the form of graphs. Based on these approaches, we propose a categorized state graph attention policy (CSGA-policy). As observed in the performance evaluation of our proposed CSGA-policy with the most well-known StarCraft II simulation environment, our proposed algorithm works well in various settings, as expected.
\end{abstract}

\section{INTRODUCTION}

In modern artificial intelligence (AI) and deep learning (DL) research [1]-[4], there are a lot of research results for developing autonomous, intelligent, and cooperative realtime strategy (RTS) game players. Among various attractive game AI research and development trends, one of major research contributions is for the design and implementation of computer game players for StarCraft II, which is the most successful world-wide RTS games [5].

In order to build AI/DL-based RTS game players, reinforcement learning (RL) and deep reinforcement learning (DRL)-based algorithms are widely used because RL/DRLbased algorithms are fundamentally for stochastic and sequential decision making in order to achieve their own goals under uncertainty observations. Therefore, the RL/DRLbased algorithms are also actively used for wireless and mobile networks [6]-[9], autonomous driving [10]-[12], robotics [13], [14], and so forth. Furthermore, in order to consider the RTS game environment which is with multiple game players, multi-agent DRL (MADRL) should be considered which is much more complicated than single-agent DRL [15], [16].

There exist a lot of research contributions in MADRL, and the corresponding algorithms can be categorized into three parts, i.e., centralized MADRL, fully decentralized MADRL and centralized training and decentralized execution (CTDE) MADRL [15]-[20].

\footnotetext{
${ }^{1}$ Won Joon Yun and Joongheon Kim are with the School of Electrical Engineering, Korea University, Seoul, Republic of Korea ywjoon95@korea.ac.kr, joongheon@korea.ac.kr

${ }^{2}$ Sungwon $\mathrm{Yi}$ is with Electronics and Telecommunications Research Institute (ETRI), Daejeon, Republic of Korea sungyi@etri.re.kr
}

In centralized-MADRL, the state information of all agents is collected in a centralized server such as cloud. Since centralized-MADRL is trained with complete state information, the performance of policy is guaranteed. However, centralized-MADRL has several challenges in reallife application. First, the relationship between the number of agents and the joint action space requires exponential complexity computation during training and execution. In addition, malfunctioned agents exist in various lives, however the computation time does not decrease. According to the aforementioned overheads, the concept of fully decentralized MADRL is re-examined.

Besides the centralized MADRL-based AI/DL algorithms, there is fully decentralized MADRL which is the opposite concept of centralized MADRL. The fully decentralized MADRL operates well without concerning the centralized MADRL problem (e.g., the exponential complexity of joint action space, or the situation of malfunctioned agents). The origin of decentralized MADRL-based algorithms is the independent Q-learning (IQL) [21], [22] which is fundamentally based on partially observable Markov decision process (POMDP) [13]. To describe the mechanism in nutshell, every single agent in IQL has its own policy and it makes decision for its own action in same environment. Because each agent has its own unique reward function, the agents take greedy actions in order to maximize their own reward without the consideration of the other agents' rewards. Therefore, it is obvious that IQL-based approach is not good for multi-agent cooperation and coordination.

In summary, there exists a dilemma between centralized MADRL and fully decentralized MADRL model. The CTDE method is proposed in order to deal with the dilemma by combining the advantage of two methods (i.e., centralized and decentralized). The CTDE method has two different procedures; 1) centralized training and 2) decentralized execution. Every agent has its own policy and make decisions with its own policy, which is similar to IQL. However, the observations and policies of all agents are trained with the centralized method for cooperation or coordination of agents. There are two main algorithms of CTDE MADRL which are based on a value decomposition network (VDN) for cooperative behaviors among multiple agents [23]. The VDN-based algorithm shows that the joint action-value function can be factorized with the summation of the all action-value functions in all agents. The proposed algorithm in [24] assumes that the joint action-value function can be factorized with monotonic mixing network (QMIX). These two algorithms, i.e., VDN and QMIX, are able to achieve 
successful factorization, moreover, they outperforms IQLbased algorithms as well-studied in [25].

When the decentralized or CTDE MADRL-based policy takes state information as an input, the state is generally composed of not only the characteristics of agents but also the partial states of other agents or other objects. If the policy is designed with deep neural network architecture based multi-layer perceptron (i.e., dense layers) [26], the units within the layers obtain every element of the given state. However, this learning mechanism is not efficient because of the unnecessary combination of state information. For that reason, this paper proposes a novel method which categorizes states. For our specific StarCraft II RTS game applications, the state categorization can be performed and three categories can be generated, i.e., the characteristics of agents, partial information of other agents, and partial information of enemies. In addition, each state is encoded to be inputs for categorized layers. The encoded states are mapped into the agents which comprise graph models, then the attention-based graph neural architectures identify the relationship among agents [27], [28].

The main contributions of this research can be summarized as following three aspects.

- First of all, a new method for categorized states is introduced based on the fact that input state information can be categorized.

- In addition, the categorized state can be mapped into agents, and then edges in graph representation can be identified via self-attention. Finally, the distributed MADRL actions can be derived by the graph representation.

- Lastly, our proposed distributed MADRL-based RTS game algorithm in this paper is evaluated in the most well-known StarCraft II RTS game environment that is called SMAC where the SMAC stands for StarCraft Multi-Agent Challenge.

The rest of this paper is organized as follows. First, we introduce the background of decentralized-MADRL in Sec. II in order to describe our proposed architecture. Then, our proposed categorized state graph attention policy (CSGApolicy) algorithm is described in Sec. III. In addition, the proposed architecture in StarCraft II is evaluated that is realtime strategy game applications in Sec. IV Lastly, Sec. V concludes this paper.

\section{RELATED WORK}

This section explains major two related neural architectures, i.e., attentive graph neural network (refer to Sec. II-A) and cooperative MADRL (refer to Sec. II-B).

\section{A. Attentive Graph Neural Architectures}

1) Attention Mechanism: An attention mechanism is widely used in various fields such as natural language processing, computer vision, distributed learning, an so forth [29]. With this attention mechanism, suppose that any types of inputs are given. Then, the input can be transformed as a context vector through encoding process. This context vector helps outputs perform well by assigning dynamic weights. Among various attention mechanisms, a scale-dot attention (i.e., query, key, and value attention) is one of well-known self-attention mechanisms. If the input $X \triangleq$ $\left\{x_{1}, x_{2}, \cdots, x_{N}\right\}$ passes through the query layer $q(\cdot)$, key layer $k(\cdot)$ and value layer $v(\cdot)$, the outputs are represented as follows.

$$
\begin{aligned}
& q(X) \triangleq\left\{q\left(x_{1}\right), q\left(x_{2}\right), \cdots, q\left(x_{N}\right)\right\} \\
& k(X) \triangleq\left\{k\left(x_{1}\right), k\left(x_{2}\right), \cdots, k\left(x_{N}\right)\right\} \\
& v(X) \triangleq\left\{v\left(x_{1}\right), v\left(x_{2}\right), \cdots, v\left(x_{N}\right)\right\} .
\end{aligned}
$$

For $\forall n \in[1, N]$ and $\forall m \in[0, N] \backslash n$, the similarity between input $x_{n}$ and $x_{m}$ can be obtained as follows,

$$
a_{n, m}=\frac{q\left(x_{n}\right) \cdot k\left(x_{m}\right)}{\sqrt{N}} * v\left(x_{m}\right) .
$$

where $a_{n, m}$ stands for attention scores.

A hard attention is a kind of stochastic attention mechanisms that is usually utilized in RL/DRL formulation. The hard attention is simpler than self-attention mechanisms because this hard attention returns only binary vector which shows whether the output is correct or not.

2) Attentive Graph Neural Architectures: A graph can be represented as $G(V, E)$ where $V$ and $E$ stand for vertices and edges, respectively. If $V$ is given and the relation among $V$ is not defined, a scale-dot attention is able to easily reveal the edges $E$ with the form of an adjacent matrix. In addition, with the hard attention of vertices $V$, the binary adjacent matrix can be obtained where the binary adjacent matrix can be implemented as a mask. Based on the scaledot attention and hard attention mechanisms, $G(V, E)$ can be finally defined.

\section{B. Cooperative $M A D R L$}

In this section, we introduce the preliminaries of the proposed architecture in terms of multi-agents' cooperation. In independent deep Q-network (I-DQN) [30], each agent $n$ is able to obtain its own observation $o_{t}^{n}$. Based on the observation, each agent decides its own action $u_{t}^{n}$ via its own policy $\pi\left(o_{t}^{n}, u_{t}^{n} ; \theta^{n}\right)$ in same environment. However, the policies of agents are all independent among them, thus the behaviors of agents are independent as well [21].

In centralized-MADRL, on the other hand, there are some policies for cooperative actions. In particular, CommNet policy makes agents be cooperative by sharing communication layers among all agents [31], [32]. Furthermore, G2ANet policy decides cooperative actions through the abstraction to graph and attention mechanism in order to represent the relationship among agents [33].

Fig. 1] shows the difference of I-DQN [21], [22], CommNet [31], [32] and G2ANet [33]. All inputs $S=$ $\left\{s^{1}, \cdots, s^{N}\right\}$ in Fig. 1 are the states those are independent. In I-DQN, it takes an independent operation for each state. Because the dense layer takes only one vector for individual computation, the actions in each agent do not consider the actions of the others. On the other hand, a CommNet-based 

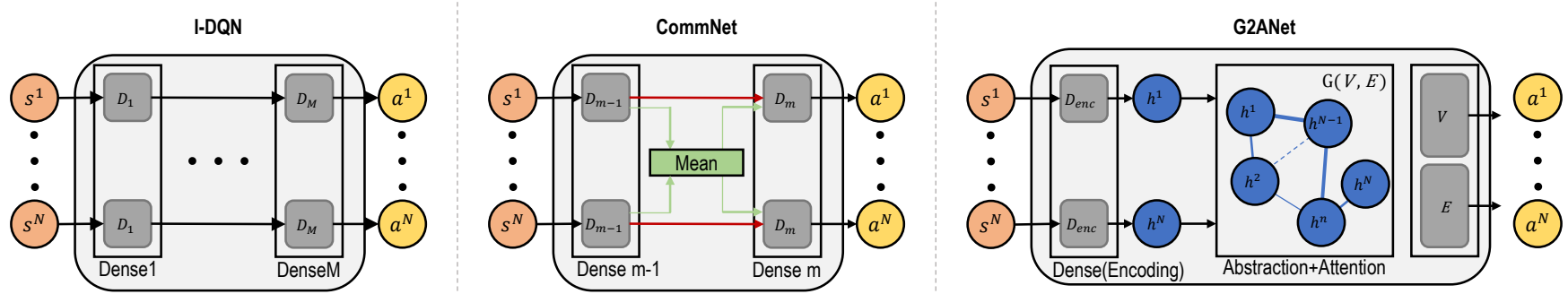

Fig. 1: Comparison among various MADRL policies.
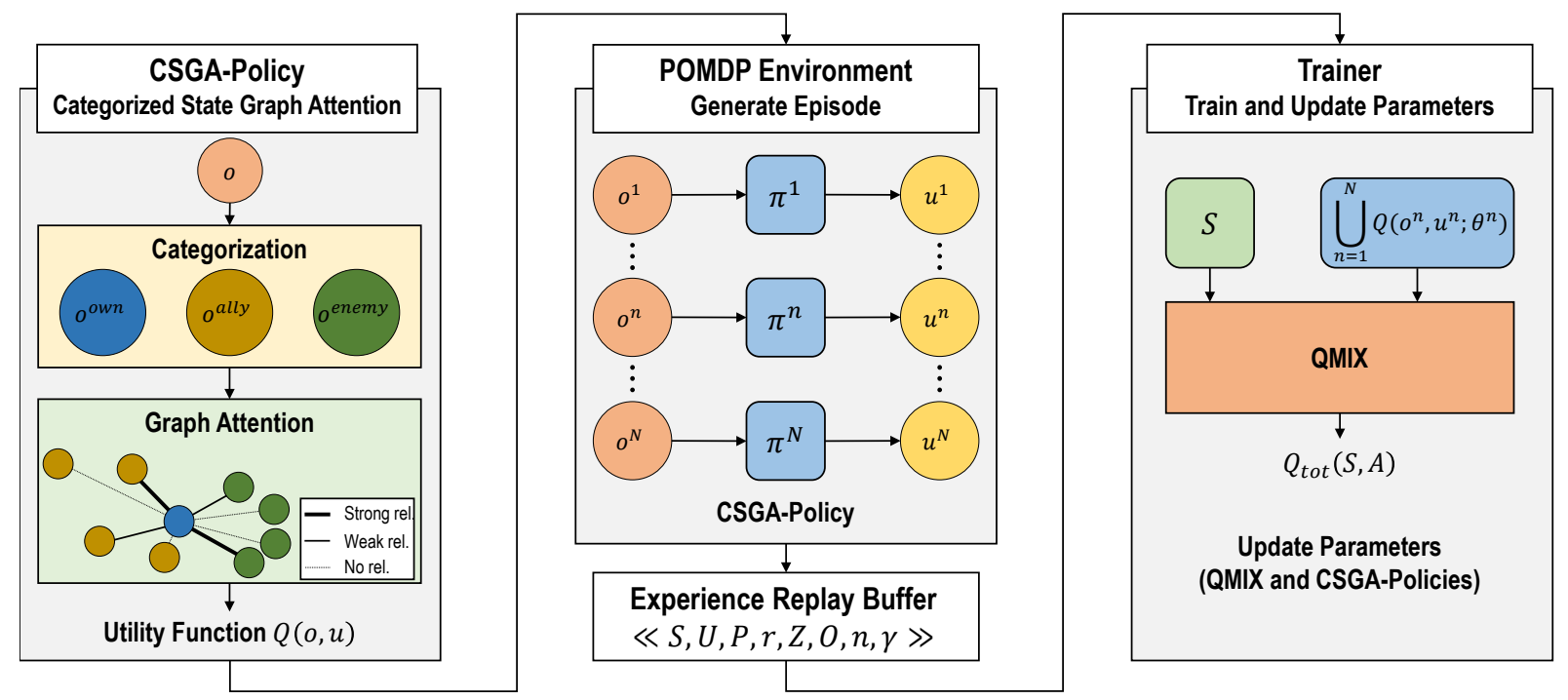

Fig. 2: Proposed MADRL-based algorithm design including CSGA-policy and QMIX neural architectures.

MADRL neural architecture [31], [32] has the communication layer that takes the mean operation of the other agents' hidden variables. For this reason, the average value of the other agents' hidden variables can affect on our considering agent's action. Therefore, we can observe that the actions are cooperative. In this CommNet-based all agents are homogeneous, thus sophisticated relationship among agents cannot be clearly represented. In case of G2ANet-based MADRL neural architectures, the agents' hidden variables are mapped into vertices $V$ via an encoding layer that is denoted as $f^{\text {enc }}$, then the vertices get attention block which can be used for calculating the weighted edges $E$. Then, $G(V, E)$ can be obtained from the attention block. Based on this graph-based structure, more complicated and sophisticated relationships among agents can be numerically identified comparing to I-DQN and CommNet based MADRL neural architectures.

In addition to CommNet-based and G2ANet-based MADRL algorithms, there exists a QMIX-based MADRL architecture which is known as semi-distributed MADRL [24]. In this QMIX-based MADRL architecture, there exists a common reward, that is defined as joint-action value function. Here, the joint action-value function is as follows,

$$
Q_{\text {total }}\left(s, u^{1}, \cdots, u^{N} ; \theta\right)=\bigcup_{n=1}^{n=N}\left\{\underset{u^{n}}{\operatorname{argmax}} Q^{n}\left(o^{n}, u^{n}\right)\right\} .
$$

During the training process, the learner selects actions $U=\left\{u^{1}, \cdots, u^{N}\right\}$ which can maximize the joint actionvalue function (5) and its own utility function $Q^{n}\left(o^{n}, u^{n}\right)$, simultaneously. For the argmax computation in (5), the QMIX MADRL neural architecture should satisfy following condition,

$$
\frac{\partial Q_{\text {total }}}{\partial Q^{n}} \geq 0, \quad \forall n \in[1, N]
$$

Aforementioned process only proceeds in training policies in a centralized manner. Aer this training phase, each QMIXbased MADRL agent can inference its own policy in a distributed manner, i.e., each agent $n$ transfers its own observation $o^{n}$ to its fine-tuned policy $\pi^{n}$ and returns cooperative actions $u^{n}$. Note that the QMIX-based MADRL algorithms can be called semi-distributed because it is centralized during training whereas it is distributed during inference.

\section{Categorized State Graph Attention Policy (CSGA-POLICY)}

In general MADRL formulations, each agent's state observation consists of the agent's unique state, other agents' partial observation, and the general state information of environment. According to the fact that StarCraft II is an RTS game, the MADRL-based game agent's state information can be categorized into agent's own observation, allies (the other agents)' partial information, and enemies' information [34]. 


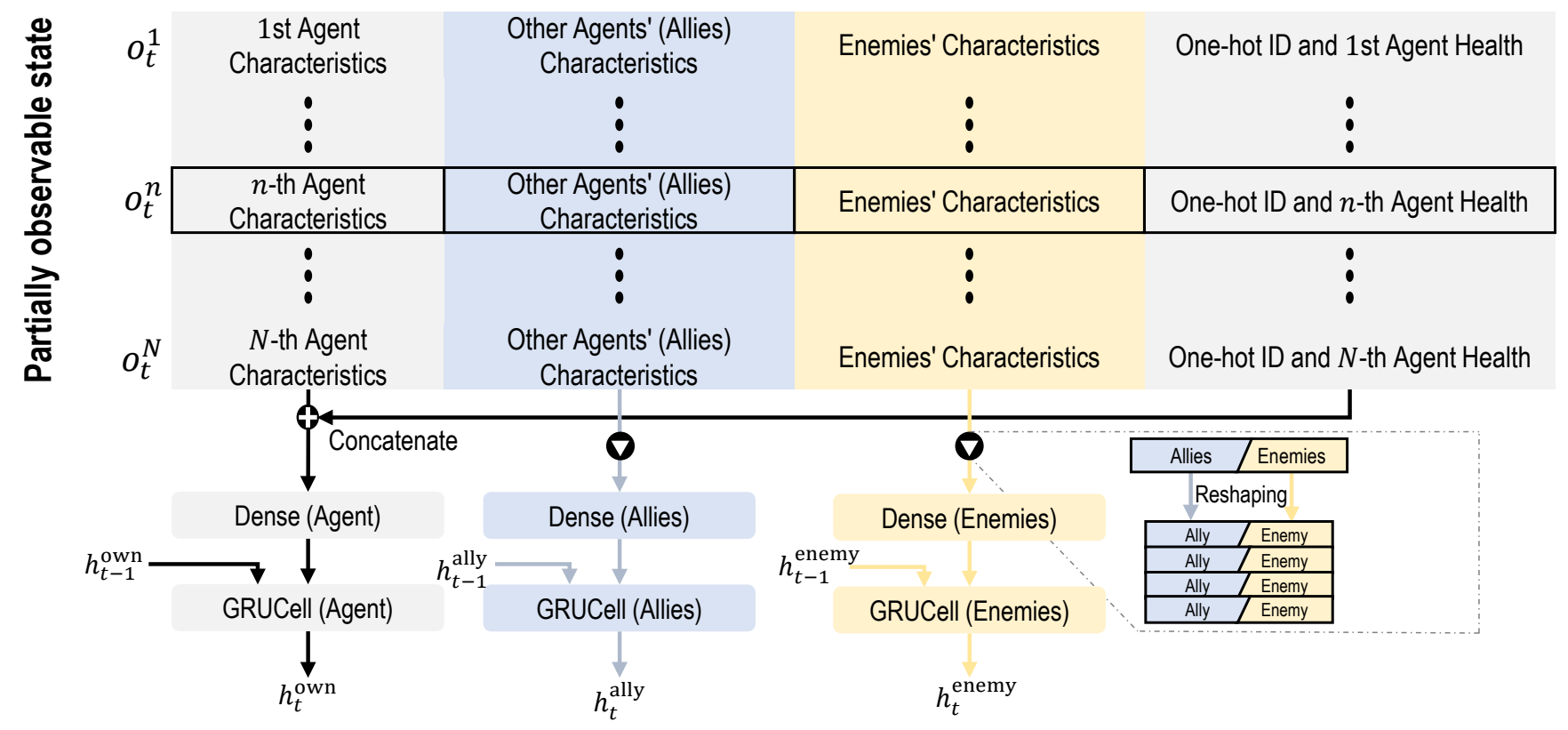

Fig. 3: Encoding and categorization processes (Step 1).

After the separation of states into aforementioned several categories, graph attention methods can be utilized for defining and learning the correlation and relationship. Our proposed MADRL-based algorithm in this paper is fundamentally based on the state categorization and attention-based graphs, thus, the algorithm is named to a Categorized State Graph Attention Policy (CSGA-Policy).

\section{A. System Model}

Our proposed MADRL neural architecture CSGA-policy is fundamentally based on deep recurrent Q-network (DRQN) [35]. To utilize the hard attention and scale-dot attention with graphs, state categorization is used. Each MADRL agent has its own CSGA-policy. In the learning phase in each agent, individual agents are trained for cooperative actions using the QMIX neural architecture. Note that the QMIX-based training is centralized whereas the inference in distributed in each MADRL agent. Fig. 3 shows the corresponding learning system model.

1) Categorization: SMAC, i.e., the StarCraft II library which provides 23 different and various combat scenarios for MADRL research [36]. There are $N$-agents and $M$ enemies in the SMAC environment. The agents can observe its own features (i.e., id, movement, health, and shield, those are denoted as $o_{n}^{\text {id }}, o_{n}^{\text {move }}, o_{n}^{\text {health }}$, and $o_{n}^{\text {shield }}$, respectively, as shown in (0.2). In addition, the agents can partially observe the features of other agents/allies (i.e., visibility, distance between agent and ally, relative $x$-position, relative $y$-position, health, and shield, those are denoted as $a_{m}^{\text {vis }}, a_{m}^{\text {dis }}$, $a_{m}^{x}, a_{m}^{y}, a_{m}^{\text {health }}$, and $a_{m}^{\text {shield }}$, respectively, as shown in (O.3) and the features of enemies (i.e., visibility, distance between agent and ally, relative $x$-position, relative $y$-position, health, and shield, those are denoted as $e_{m}^{\text {vis }}, e_{m}^{\text {dis }}, e_{m}^{x}, e_{m}^{y}, e_{m}^{\text {health }}$, and $e_{m}^{\text {shield }}$, respectively, as shown in (O.4).
Each agent $n$ with the observation $o^{n}$ in $\mathrm{O} .1$ can be represented as $\mathrm{O} .1-\mathrm{O} .4$,

$$
\begin{aligned}
& o^{n} \triangleq\left\{o^{n, \text { own }}, o^{n, \text { ally }}, o^{n, \text { enemy }}\right\}, \\
& o^{n, \text { own }} \triangleq\left\{o_{n}^{\text {id }}, o_{n}^{\text {move }}, o_{n}^{\text {health }}, o_{n}^{\text {shield }}\right\},(\mathrm{O} .1) \\
& o^{n, \text { ally }} \triangleq \bigcup_{m \neq n, m \geq 1}^{m=N}\left\{a_{m}^{\text {vis }}, a_{m}^{\text {dis }}, a_{m}^{x}, a_{m}^{y}, a_{m}^{\text {health }}, a_{m}^{\text {shield }}\right\}(\mathrm{O} .3) \\
& o^{n, \text { enemy }} \triangleq \bigcup_{l \neq n, l \geq 1}^{l=N}\left\{e_{l}^{\text {vis }}, e_{l}^{\text {dis }}, e_{l}^{x}, e_{l}^{y}, e_{l}^{\text {health }}, e_{l}^{\text {shield }}\right\},
\end{aligned}
$$

where $a_{m}^{\text {feature }}$ and $e_{l}^{\text {feature }}$ stand for feature of $m$-th ally and $l$-th enemy. The $o^{n}$ can be defined as the combination of following three, i.e., $o^{n \text {,own }}, o^{n \text {, ally }}$, and $o^{n \text {,enemy }}$, as shown in O.1. Fig. 3 illustrates the information that agents observe. As illustrated in Fig. 3, when $N-1$ allies and $L$ enemies exist, $o^{n, \text { ally }}$ and $o^{n \text {,enemy }}$ are transformed to ally matrix $\mathcal{A}^{n}$ (size: $(N-1) \times \operatorname{dim}($ feature) ) and enemy matrix $\mathcal{E}^{n}$ (size: $L \times \operatorname{dim}$ (feature)), respectively, where $\operatorname{dim}(k)$ is defined as a function to obtain the dimension of given vector $k$. At time $t, \mathcal{A}_{t}^{\mathrm{enc}, n}$ and $\mathcal{E}_{t}^{\mathrm{enc}, n}$ are defined as the results of encoding layer when the inputs are $\mathcal{A}^{n}$ and $\mathcal{E}^{n}$, respectively. The $\mathcal{A}_{t}^{\text {enc, } n}$ and hidden layer parameter $h_{t-1}^{\text {ally }}$ will be the input of ally-RNN, and similarly, $\mathcal{E}_{t}^{\text {enc, } n}$ and hidden layer $h_{t-1}^{\text {enemy }}$ will be the input of enemy-RNN, as well.

2) Graph Attention: There are two major methods for graph attentions, i.e., hard attention and scale-dot attention where the hard attention is for representing the relationship among nodes with 0 or 1 whereas the scale-dot attention is for determining the weights over the graph edges. The encoding process in Fig. 3 takes three different types of input components, i.e., agent's its own hidden variable $h_{t}^{\text {own }}$, allies' 


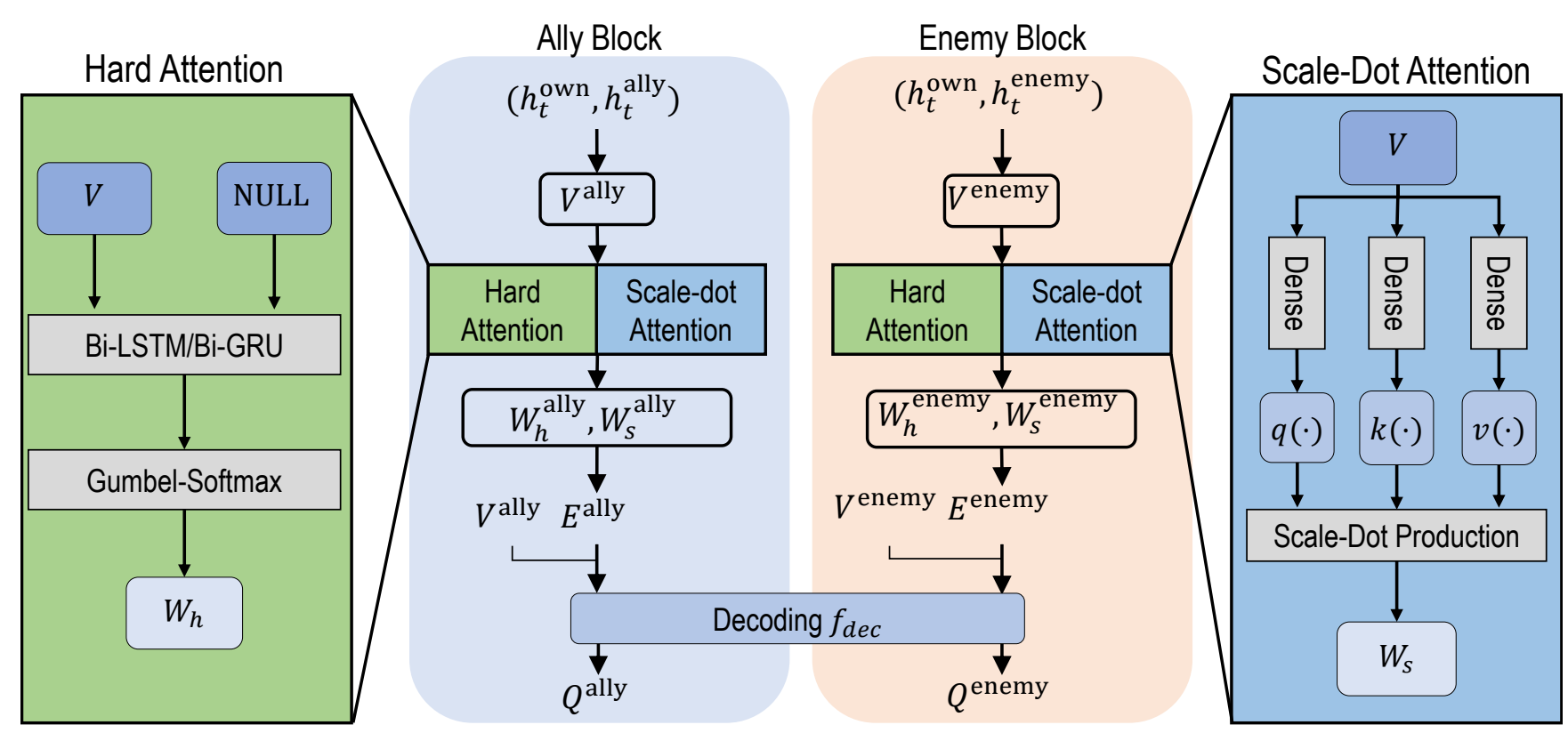

Fig. 4: Attention and decoding processes (Step 2).

their own hidden variable $h_{t}^{\text {ally }}$, and enemies' hidden variable $h_{t}^{\text {enemy }}$.

Fig. 4 illustrates the MADRL learning computational procedures for allies and enemies. The proposed MADRLbased algorithm gathers the information after state categorization, and separates the results into allies' information and enermies' information. After that, the results will be the inputs for Ally Block and Enemy Block in Fig. 4.

In order to obtain own-allies graph and own-enemies graph, the propose method obtains $V^{\text {ally }}$ and $V^{\text {enemy }}$ at first, where the $V^{\text {ally }}$ and $V^{\text {enemy }}$ are defined as the $(N-1) \times$ dim (RNN) matrix which is $N-1$ times replication of $h_{t}^{\text {own }}$ and the $L \times \operatorname{dim}(\mathrm{RNN})$ matrix which is $L$ times replication of $h_{t}^{\text {enemy }}$, respectively. Then, these $V^{\text {ally }}$ and $V^{\text {enemy }}$ can be mapped to $N-1$ vertices and $L$ vertices, respectively. Suppose that NULL ${ }^{\text {ally }}$ and NULL $^{\text {enemy }}$ are the matrices where all elements are zero with the sizes of $(N-1) \times \operatorname{dim}(\mathrm{RNN})$ and $L \times \mathrm{dim}(\mathrm{RNN})$.

Based on these obtained $V^{\text {ally }}, V^{\text {enemy }}$, NULL ${ }^{\text {ally }}$, and NULL ${ }^{\text {enemy }}$, following computation can be done in order to get the weight vectors $W_{h}^{\text {ally }}$ (size: $N-1$ ) and $W_{h}^{\text {enemy }}$ (size: $L$ ) where the values of vectors are 0 and 1 ,

$$
\begin{aligned}
W_{h}^{\text {ally }} & =\text { G-softmax }\left(B i G R U\left(V^{\text {ally }}, \mathrm{NULL}^{\text {ally }}\right)\right) \\
W_{h}^{\text {enemy }} & =\text { G-softmax }\left(B i G R U\left(V^{\text {enemy }}, \text { NULL }^{\text {enemy }}\right)(\right.
\end{aligned}
$$

where G-softmax( $(\cdot)$ and BiGRU(.) stand for gumbelsoftmax [37] and bidirectional GRU cell [38], respectively. After this computation, the attention scores $A t t^{\text {ally }}$ and $A t t^{\text {enemy }}$ should be obtained via $V^{\text {ally }}$ (for own-ally) and $V^{\text {enemy }}$ (for own-enemy) (1)-(4), respectively. Finally, soft weights $W_{s}^{\text {ally }}$ and $W_{s}^{\text {ehemy }}$ can be obtained applying Softmax function to the attention scores $A t t^{\text {ally }}$ and $A t t^{\text {enemy }}$. Now, the weighted adjacency matrices $E^{\text {ally }}$, $E^{\text {enemy }}$ can be computed using element-wise product for the given $\left(W_{h}^{\text {ally }}, W_{s}^{\text {ally }}\right)$ and ( $\left.W_{h}^{\text {enemy }}, W_{s}^{\text {enemy }}\right)$.

Lastly, based on these results, the utility function as the results of decoding layers where the inputs are $G\left(V^{\text {ally }}, E^{\text {ally }}\right)$ and $G\left(V^{\text {enemy }}, E^{\text {enemy }}\right)$ is as follows,

$$
\begin{aligned}
Q^{\text {ally }}\left(o^{n}, u^{n}\right) & =\frac{1}{N-1} f^{\text {dec }}\left(G\left(V^{\text {ally }}, E^{\text {ally }}\right)\right) \\
Q^{\text {enemy }}\left(o^{n}, u^{n}\right) & =\frac{1}{L} f^{\text {dec }}\left(G\left(V^{\text {enemy }}, E^{\text {enemy }}\right)\right)
\end{aligned}
$$

where $f^{\text {dec }}$ stands for the functional representation of decoding layers and $u^{n}$ represents the action of $n$-th agent. Our final result $Q\left(o^{n}, u^{n}\right)$ can be obtained by taking softmax function for $Q^{\text {ally }}\left(o^{n}, u^{n}\right)$ and $Q^{\text {enemy }}\left(o^{n}, u^{n}\right)$, and also adding weights.

\section{Performance Evaluation}

This section consists of simulation setup (refer to Sec. IVA and results (refer to Sec. IV-B), respectively.

\section{A. Simulation Setup}

Our simulation is performed in Windows operating systems and this MADRL is implemented with Python with open library Pytorch/Pysc2/SMAC. Among various maps provided by SMAC, our simulation-based evaluation is performed using the scenarios with 3 marines (called Simulation-3m) and 8 marines (called Simulation-8m) where the level/difficulty of the game is set to 7 (i.e., very hard). For exploit-and-exploration, $\epsilon$-greedy is used where $\epsilon=0.3$ [39]. As illustrated in Fig. 2, actor and critic neural architectures are CSGA-policy and QMIX, respectively. The corresponding training is conducted with temporal difference [40] where the number of training iteration is 100,000 . 


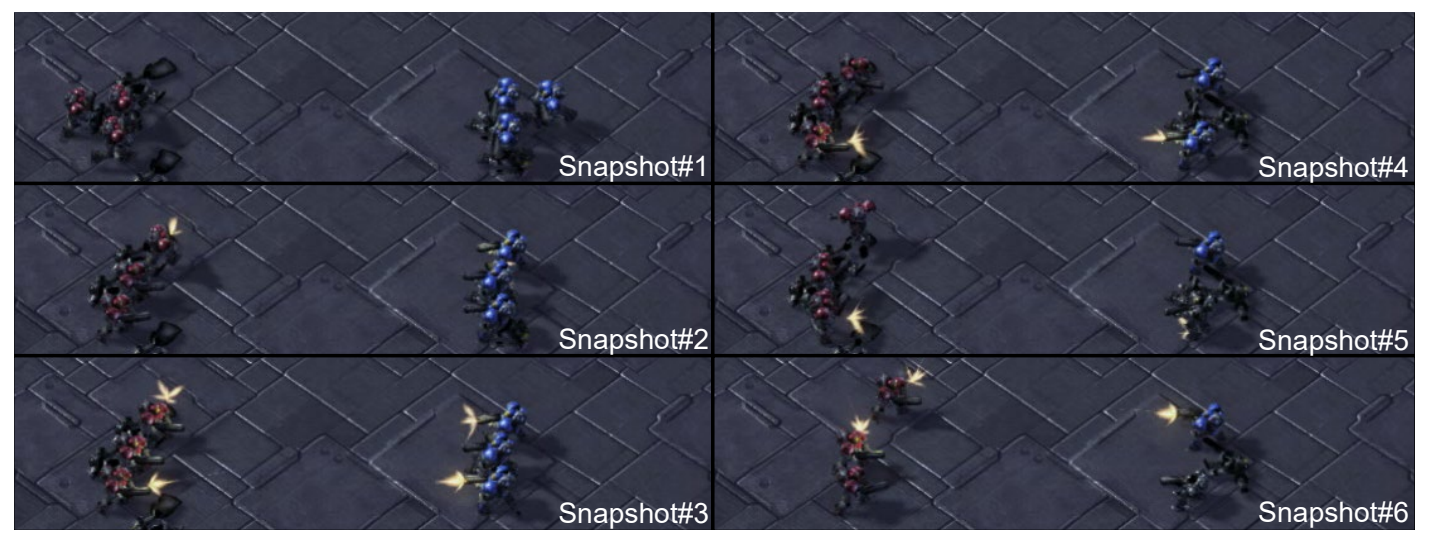

Fig. 5: MADRL performance evaluation results with Simulation-3m (3 marines vs. 3 marines).

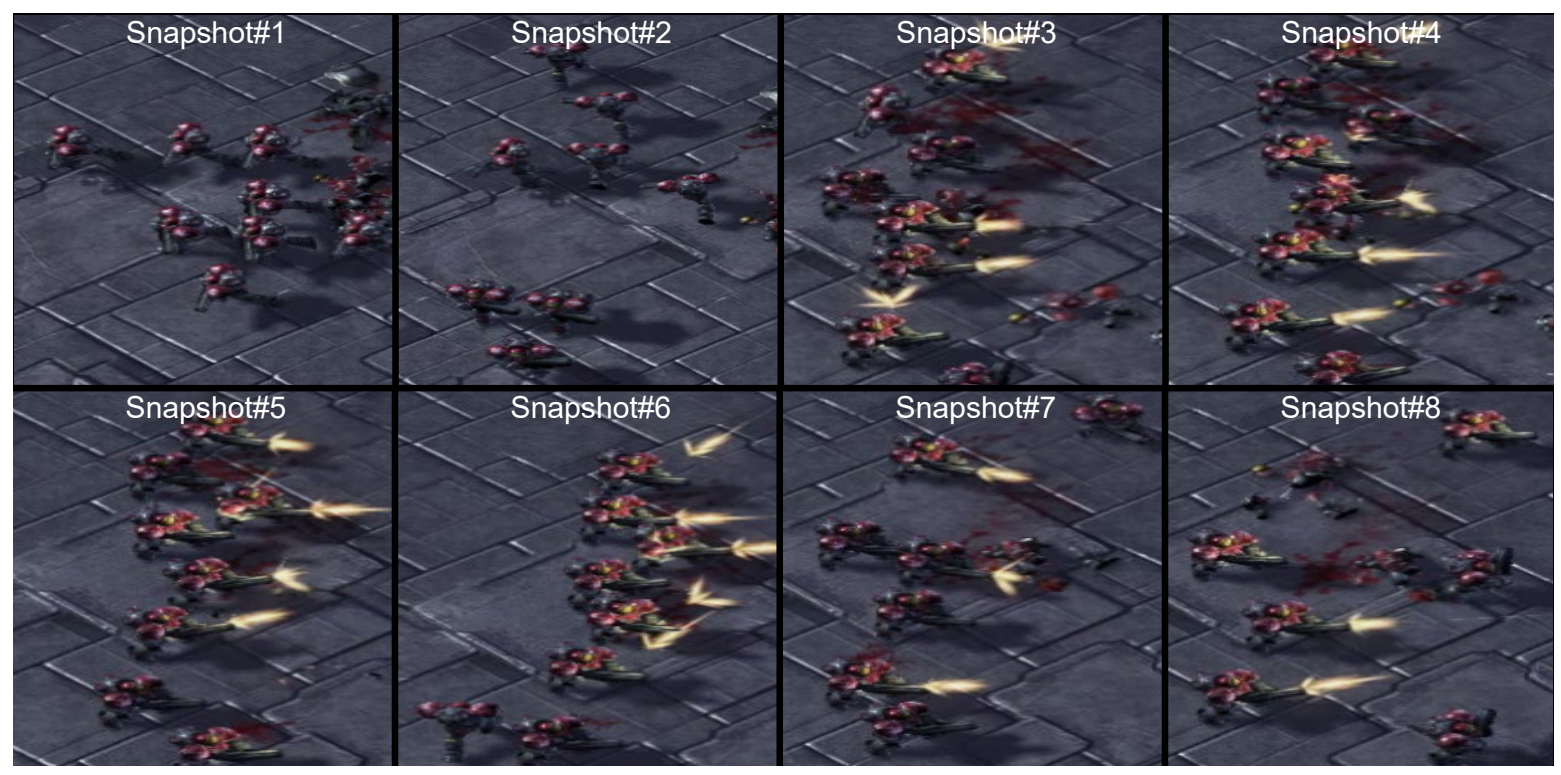

Fig. 6: MADRL performance evaluation results with Simulation-8m (8 marines vs. 8 marines).

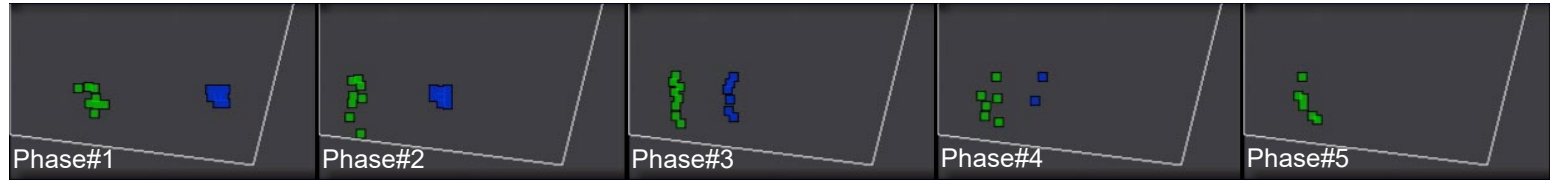

Fig. 7: The locations of agents in Simulation-8m (green: agents, blue: enemies).

\section{B. Simulation Results}

The SMAC simulation results in terms of Simulation$3 \mathrm{~m}$ and Simulation-8m are presented in Fig. 5. Fig. 6, and Fig.7 In the results, red units and blue units are for our ally agents and enemy agents (where the enemy is set to the level 7 which means very hard), respectively. The performance evaluation results for Simulation-3m and Simulation-8m are in following Sec. IV-B.1 and Sec. IV-B.2, respectively. Furthermore, the demo video is available on [41].

1) Simulation Results (3 marines): The six snapshots in Fig. 5 are time-series representations of multi-agent actions for the case of Simulation-3m. In Fig. 5. Snapshot \#1 shows the situation before combat. Snapshots from \#2 to \#4 is for focused shooting and thus we can observed that one enemy is eliminated. In Snapshot\#5, one of our ally marines moves up because its own health condition is not good (thus, it wants to avoid the combat in order to keep its own health condition). In Snapshot \#6, the ally marine who moved up in Snapshot \#5 is back and attacks the enemies, and finally all enemies are eliminated. In our entire 20 simulations, we confirm that our ally marines are all alive (i.e., winning rate: $100 \%)$.

2) Simulation Results (8 marines): The eight snapshots in Fig. 6 are time-series representations of multi-agent actions for the case of Simulation-8m. In addition, Fig. 7 shows the positions of ally units and enemy units. During the early stages (i.e., the Snapshots from \#1 to \#3 in Fig. 6 and the 
Phases from \#1 to \#2 in Fig. 77, our ally marines move back, arrange combat formation, and lure the enemy units into our desired position. In the middle stages (i.e., the Snapshots from \#4 to \#8 in Fig. 6 and the Phases from \#3 to \#4 in Fig. 7), our ally units attack the enemy units, and the ally units who health conditions are not good move back. Lastly, we can observe that all enemy units are eliminated in the Phase \#5 in Fig. 7 .

\section{CONCluding Remarks}

This paper proposes a novel multi-agent deep reinforcement learning (MADRL) algorithm that controls multiple agents in real-time strategy (RTS) games. Among them, our proposed MADRL-based algorithm is fundamentally based on distributed computation and it is the combination of categorized state graph attention policy (CSGA-policy) and QMIX neural architectures. The CSGA-policy is the for state categorization and graph attention where the graph attention is used for learning the relationship among agents. The training is performed with QMIX which is centralized during training whereas it is distributed among agents during inference for individual action taking. Our performance evaluation results verify that the proposed algorithm works well in StarCraft II environment with various settings.

\section{ACKNOWLEDGMENT}

This research was supported in part by Electronics and Telecommunications Research Institute (ETRI) grants 19YE1410 and Air Force Office of Scientific Research (AFOSR) grants FA2386-19-1-4020. S. Yi and J. Kim are the corresponding authors of this paper.

\section{REFERENCES}

[1] D. Perez-Liebana, J. Liu, A. Khalifa, R. D. Gaina, J. Togelius, and S. M. Lucas, "General video game AI: A multitrack framework for evaluating agents, games, and content generation algorithms," IEEE Transactions on Games, vol. 11, no. 3, pp. 195-214, September 2019.

[2] J. Park, S. Samarakoon, A. Elgabli, J. Kim, M. Bennis, S.-L. Kim, and M. Debbah, "Communication-efficient and distributed learning over wireless networks: Principles and applications," arXiv preprint arXiv:2008.02608, August 2020.

[3] D. Kim, J. Kim, J. Kwon, and T. Kim, "Depth-controllable very deep super-resolution network," in Proceedings of the IEEE International Joint Conference on Neural Networks (IJCNN), Budapest, Hungary, July 2019, pp. 1-8.

[4] S. Ahn, J. Kim, E. Lim, W. Choi, A. Mohaisen, and S. Kang, "ShmCaffe: A distributed deep learning platform with shared memory buffer for HPC architecture," in Proceedings of the IEEE International Conference on Distributed Computing Systems (ICDCS), Vienna, Austria, July 2018, pp. 1118-1128.

[5] L. Wang, Y. Zeng, B. Chen, Y. Pan, and L. Cao, "Team recommendation using order-based fuzzy integral and NSGA-II in StarCraft," IEEE Access, vol. 8, pp. 59559-59570, March 2020.

[6] D. Kwon, J. Jeon, S. Park, J. Kim, and S. Cho, "Multiagent DDPGbased deep learning for smart ocean federated learning IoT networks," IEEE Internet of Things Journal, vol. 7, no. 10, pp. 9895-9903, October 2020.

[7] D. Kwon, J. Kim, D. A. Mohaisen, and W. Lee, "Self-adaptive power control with deep reinforcement learning for millimeter-wave Internetof-vehicles video caching," Journal of Communications and Networks, vol. 22, no. 4, pp. 326-337, August 2020.

[8] M. Choi, A. No, M. Ji, and J. Kim, "Markov decision policies for dynamic video delivery in wireless caching networks," IEEE Transactions on Wireless Communications, vol. 18, no. 12, pp. 57055718, December 2019.
[9] F. Jiang, C. Zheng, D. Gao, X. Zhang, W. Liu, Y. Cheng, C. Hu, and J. Peng, "A novel multi-agent cooperative reinforcement learning method for home energy management under a peak power-limiting," in Proceedings of the IEEE International Conference on Systems, Man, and Cybernetics (SMC), Toronto, ON, Canada, October 2020, pp. 350355.

[10] M. Shin and J. Kim, "Adversarial imitation learning via random search," in Proceedings of the IEEE International Joint Conference on Neural Networks (IJCNN), Budapest, Hungary, July 2019, pp. 1-8.

[11] M. Shin and J. Kim, "Randomized adversarial imitation learning for autonomous driving," in Proceedings of the Twenty-Eighth International Joint Conference on Artificial Intelligence (IJCAI), Macau, China, August 2019, pp. 4590-4596.

[12] J. Källström and F. Heintz, "Agent coordination in air combat simulation using multi-agent deep reinforcement learning," in Proceedings of the IEEE International Conference on Systems, Man, and Cybernetics (SMC), Toronto, ON, Canada, October 2020, pp. 2157-2164.

[13] D. K. Grady, M. Moll, and L. E. Kavraki, "Extending the applicability of POMDP solutions to robotic tasks," IEEE Transactions on Robotics, vol. 31, no. 4, pp. 948-961, August 2015.

[14] J. Kober and J. Peters, "Reinforcement learning in robotics: A survey," in The International Journal of Robotics Research, October 2013, vol. 32, no. 11, pp. $1238-1274$.

[15] D. Kwon and J. Kim, "Multi-agent deep reinforcement learning for cooperative connected vehicles," in Proceedings of the IEEE Global Communications Conference (GLOBECOM), Waikoloa, HI, USA, December 2019, pp. 1-6.

[16] L. Busoniu, R. Babuska, and B. De Schutter, "A comprehensive survey of multiagent reinforcement learning," IEEE Transactions on Systems, Man, And Cybernetics, no. 2, February 2008.

[17] D. Horgan, J. Quan, D. Budden, G. Barth-Maron, M. Hessel, H. Van Hasselt, and D. Silver, "Distributed prioritized experience replay," arXiv preprint arXiv:1803.00933, 2018.

[18] P. Henderson, R. Islam, P. Bachman, J. Pineau, D. Precup, and D. Meger, "Deep reinforcement learning that matters," arXiv preprint arXiv:1709.06560, 2017.

[19] P. Xuan and V. Lesser, "Multi-agent policies: from centralized ones to decentralized ones," in Proceedings of The First International Joint Conference on Autonomous Agents and Multiagent Systems (AAMAS), July 2002, pp. 1098-1105.

[20] T. Salimans, J. Ho, X. Chen, S. Sidor, and I. Sutskever, "Evolution strategies as a scalable alternative to reinforcement learning," arXiv preprint arXiv:1703.03864, 2017.

[21] M. Tan, "Multi-agent reinforcement learning: Independent versus cooperative agents," in Proceedings of the Tenth International Conference on Machine Learning (ICML), San Francisco, CA, USA, June 1993, pp. 330-337.

[22] V. Mnih, K. Kavukcuoglu, D. Silver, A. Graves, I. Antonoglou, D. Wierstra, and M. Riedmiller, "Playing Atari with deep reinforcement learning," arXiv preprint arXiv:1312.5602, December 2013.

[23] P. Sunehag, G. Lever, A. Gruslys, W. M. Czarnecki, V. Zambaldi, M. Jaderberg, M. Lanctot, N. Sonnerat, J. Z. Leibo, K. Tuyls, and T. Graepel, "Value-decomposition networks for cooperative multiagent learning based on team reward," in Proceedings of the International Conference on Autonomous Agents and Multiagent Systems (AAMAS), Stockholm, Sweden, July 2018, pp. 2085-2087.

[24] T. Rashid, M. Samvelyan, C. S. de Witt, G. Farquhar, J. N. Foerster, and S. Whiteson, "QMIX: Monotonic value function factorisation for deep multi-agent reinforcement learning," in Proceedings of the ThirtySeventh International Conference on Machine Learning (ICML), Vienna, Austria, July 2018, pp. 4292-4301.

[25] M. Zhou, Y. Chen, Y. Wen, Y. Yang, Y. Su, W. Zhang, D. Zhang, and J. Wang, "Factorized q-learning for large-scale multi-agent systems," in Proceedings of the First ACM International Conference on Distributed Artificial Intelligence (DAI), Beijing, China, October 2019, pp. 1-7.

[26] Y. J. Mo, J. Kim, J. Kim, A. Mohaisen, and W. Lee, "Performance of deep learning computation with tensorflow software library in gpu-capable multi-core computing platforms," in Proceedings of the IEEE International Conference on Ubiquitous and Future Networks (ICUFN), Milan, Italy, July 2017, pp. 240-242.

[27] F. Scarselli, M. Gori, A. C. Tsoi, M. Hagenbuchner, and G. Monfardini, "The graph neural network model," IEEE Transactions on Neural Networks, vol. 20, no. 1, pp. 61-80, January 2008. 
[28] C. Zhang, D. Song, C. Huang, A. Swami, and N. V. Chawla, "Heterogeneous graph neural network," in Proceedings of the 25th ACM SIGKDD International Conference on Knowledge Discovery \& Data Mining, August 2019, pp. 793-803.

[29] A. Vaswani, N. Shazeer, N. Parmar, J. Uszkoreit, L. Jones, A. N. Gomez, L. Kaiser, and I. Polosukhin, "Attention is all you need," in Proceedings of the Advances in Neural Information Processing Systems (NIPS), vol. 30, Long Beach, CA, USA, December 2017, pp. 5998-6008.

[30] J. N. Foerster, Y. M. Assael, N. De Freitas, and S. Whiteson, "Learning to communicate with deep multi-agent reinforcement learning," in Proceedings of the 30th International Conference on Neural Information Processing Systems (NIPS), December 2016, pp. 2145-2153.

[31] S. Jung, W. J. Yun, J. Kim, and J.-H. Kim, "Infrastructure-assisted cooperative multi-UAV deep reinforcement energy trading learning for big-data processing," in Proceedings of the IEEE International Conference on Information Networking (ICOIN), Jeju Island, Republic of Korea, January 2021.

[32] M. Shin, D. Choi, and J. Kim, "Cooperative management for PV/ESSenabled electric vehicle charging stations: A multiagent deep reinforcement learning approach," IEEE Transactions on Industrial Informatics, vol. 16, no. 5, pp. 3493-3503, May 2020.

[33] Y. Liu, W. Wang, Y. Hu, J. Hao, X. Chen, and Y. Gao, "Multi-agent game abstraction via graph attention neural network," in Proceedings of the Thirty-Fourth AAAI Conference on Artificial Intelligence (AAAI), New York, NY, USA, February 2020, pp. 7211-7218.

[34] O. Vinyals, T. Ewalds, S. Bartunov, P. Georgiev, A. S. Vezhnevets, M. Yeo, A. Makhzani, H. Küttler, J. P. Agapiou, J. Schrittwieser, J. Quan, S. Gaffney, S. Petersen, K. Simonyan, T. Schaul, H. van Hasselt, D. Silver, T. P. Lillicrap, K. Calderone, P. Keet, A. Brunasso, D. Lawrence, A. Ekermo, J. Repp, and R. Tsing, "StarCraft II: A new challenge for reinforcement learning," arXiv preprint arXiv:1708.04782, August 2017.

[35] M. Hausknecht and P. Stone, "Deep recurrent Q-learning for partially observable MDPs," arXiv preprint arXiv:1507.06527, 2015.

[36] M. Samvelyan, T. Rashid, C. S. De Witt, G. Farquhar, N. Nardelli, T. G. Rudner, C.-M. Hung, P. H. Torr, J. Foerster, and S. Whiteson, "The starcraft multi-agent challenge," arXiv preprint arXiv:1902.04043, February 2019.

[37] E. Jang, S. Gu, and B. Poole, "Categorical reparameterization with Gumbel-Softmax," in Proceedings of the Fifth International Conference on Learning Representations (ICLR), Toulon, France, July 2017.

[38] Y. Deng, L. Wang, H. Jia, X. Tong, and F. Li, "A sequence-to-sequence deep learning architecture based on bidirectional GRU for type recognition and time location of combined power quality disturbance," IEEE Transactions on Industrial Informatics, vol. 15, no. 8, pp. 4481-4493, August 2019.

[39] S. Levine and V. Koltun, "Guided policy search," in Proceedings of the International Conference on Machine Learning (ICML), Atlanta, GA, USA, June 2013, pp. 1-9.

[40] G. Tesauro, "Temporal difference learning and TD-gammon," Communications of the ACM, vol. 38, no. 3, pp. 58-68, March 1995.

[41] W. J. Yun, S. Yi, and J. Kim, "Multi-agent deep reinforcement learning using attentive graph neural architectures for real-time strategy games," https://sites.google.com/view/smc-2021-madrl-csga 2021. 